\title{
WAVES PRODUCED BY A PULSATING SOURCE TRAVELLING BENEATH A FREE SURFACE*
}

BT

\author{
H. 8. TAN \\ University of Notre Dame**
}

Summary. The propagation of surface waves induced by a pulsating source travelling beneath a free surface is investigated by introducing Rayleigh's dissipative force. By defining a disturbance velocity potential $R \varphi(x, y)$ exp $(i \omega t)$, it is shown that $\varphi(x, y)$ has to satisfy throughout the flow field not only the Laplace equation, but also a differential equation resulting from the analytic extension of the free surface boundary condition. The solution is obtained in the form

$$
\varphi(x, y)=f(z)+g(\bar{z}), \quad z=x+i y,
$$

i.e., $\varphi(x, y)$ is a complex harmonic function of $x, y$ but is not an analytic function of $z$. The wave propagation is found to depend on a parameter $\tau$, which is the ratio of the pulsation frequency $\omega$ of the source strength to the fundamental frequency $\omega_{0}$ of the surface wave produced by a constant strength source travelling at the same speed. The case $\tau=0$, corresponding to the travelling source of constant strength, does give a single undamped harmonic wave train on the downstream side, of wave length $2 \pi c^{2} / g$, (or frequency $\omega_{0}$ ), as is expected. For $0<\tau<1 / 4$, there are four, and for $\tau>1 / 4$, two undamped harmonic wave trains of different wave lengths on the downstream side. It is further observed that there exists a critical frequency at $\tau=1 / 4$, at which frequency resonance phenomena occur. Thus violent disturbance is predicted at $\tau=1 / 4$ by the present analysis. No disturbance is found to propagate infinitely upstream. This result evidently justifies the imposition of an asymptotic upstream condition of "vanishing disturbance at infinity" to replace the effect of the dissipative force in rendering the solution unique. ${ }^{1}$

1. Velocity potential. Consider a two dimensional incompressible fluid of infinite depth and extent, beneath whose free surface a point source is travelling at a constant speed $c$. In an $(x, y)$ coordinate system that moves with the source, and under the hypothesis that the resulting fluid motion is irrotational, one can define an absolute disturbance velocity potential $\Phi(x, y, t)$ through the differential equation

$$
\Phi_{x x}+\Phi_{y y}=0
$$

and the boundary condition over the free surface $Y(y=0)$

i.e.

$$
\begin{aligned}
& \Phi_{t}-c \Phi_{x}=-g Y, \\
& Y_{\imath}-c Y_{x}=\Phi_{y},
\end{aligned}
$$

$$
\Phi_{\imath \imath}-2 c \Phi_{\imath x}+c^{2} \Phi_{x x}+g \Phi_{y}=0 .
$$

When the strength of the travelling source fluctuates harmonically, we may write the velocity potential in the form:

*Received April 16, 1956; revised manuscript received July 16, 1956.

**Now at University of Detroit. 


$$
\Phi(x, y, t)=\operatorname{R\varphi }(x, y) \exp (i \omega t)
$$

and obtain the following differential system for $\varphi$ :

$$
\begin{gathered}
\varphi_{x x}+\varphi_{y y}=0, \\
\nu \varphi_{y}-2 i \tau \nu \varphi_{x}-\nu^{2} \varphi+\tau^{2} \varphi_{x x}=0, \quad y=0,
\end{gathered}
$$

where

$$
\tau=\omega c / g, \quad \nu=\omega^{2} / g .
$$

It is a well known fact that the solution of this problem is indeterminate, as free harmonic surface wave trains of appropriate wave lengths can be added without disturbing either the differential equation or the boundary condition. This indeterminacy is removed by introducing an artificial dissipative force $-\mu \nabla \Phi$ and then letting $\mu$ approach zero in the result. This artifice was first introduced by Rayleigh ${ }^{2}$, and has since been successfully employed by various investigators ${ }^{3,4,5}$ for studying surface waves set up by moving sources and sinks of constant strengths.

By adopting this scheme, the differential equations (1) and (4) are not affected, but the boundary conditions (2) and (5) are replaced by

$$
\begin{gathered}
\Phi_{\iota t}-2 c \Phi_{\iota x}+c^{2} \Phi_{x x}+\mu \Phi_{\iota}-\mu c \Phi_{x}+g \Phi_{y}=0, \\
\nu \varphi_{y}-2 i \tau \nu(1-i \beta) \varphi_{x}-\nu^{2}(1-2 i \beta) \varphi+\tau^{2} \varphi_{x x}=0,
\end{gathered}
$$

respectively for $y=0$, where $\beta=\mu / 2 \omega$.

Let us now write the spatial part of our velocity potential (with moving source located at $\zeta=\xi+i \eta$ ) in the form

$$
\varphi=\ln |z-\zeta|-\ln |z-\xi|+G(x, y ; \xi, \eta), \quad z=x+i y,
$$

where $G(x, y ; \xi, \eta)$ is harmonic on the lower half plane. Noting that on $y=0$,

$$
\begin{aligned}
& \frac{\partial}{\partial y} \ln |z-\zeta|=-\frac{\partial}{\partial y} \ln |z-\xi|=\Im(z-\xi)^{-1}, \\
& \frac{\partial^{n}}{\partial x^{n}} \ln |z-\zeta|=\frac{\partial^{n}}{\partial x^{n}} \ln |z-\xi|,
\end{aligned}
$$

and substituting (8) into (7), we obtain the following differential equation for $G$ on $y=0$ :

$$
\nu G_{\nu}-2 i \tau \nu(1-i \beta) G_{x}-\nu^{2}(1-2 i \beta) G+\tau^{2} G_{x x}=2 \nu \frac{\partial^{\bullet}}{\partial y} \ln |z-\xi| .
$$

As both sides of (10) involve functions that are harmonic in the lower half plane, (10) may be required to hold for the entire lower half plane by analytic extension. It can indeed easily be shown that a general solution of the Laplace equation is given by $f(z)+g(\bar{z})$.

Now we have the well known relation

$$
(z-\tilde{\zeta})^{-1}=i \int_{0}^{\infty} \exp [-i \kappa(z-\tilde{\xi})] d \kappa, \quad y+\eta<0
$$

from which the following expression can readily be deduced: 


$$
\begin{aligned}
2 \nu \frac{\partial}{\partial y} \ln |z-\xi|=-\nu \int_{0}^{\infty} \exp [\kappa(y+\eta)]\left\{\exp \left[i_{\kappa}(x-\xi)\right]\right. & \\
& \left.+\exp \left[-i_{\kappa}(x-\xi)\right]\right\} d \kappa .
\end{aligned}
$$

Combining equations (12) and (10), we arrive at

$$
\begin{aligned}
G=\nu \int_{0}^{\infty} \frac{\exp [\kappa(y+\eta)]}{\tau^{2} \kappa^{2}+[2 \tau(1-i \beta)-1] \nu \kappa+\nu^{2}(1-2 i \beta)} \exp [-i \kappa(x-\xi)] d \kappa \\
\quad+\nu \int_{0}^{\infty} \frac{\exp [\kappa(y+\eta)]}{\tau^{2} \kappa^{2}-[2 \tau(1-i \beta)+1] \nu \kappa+\nu^{2}(1-2 i \beta)} \exp [i \kappa(x-\xi)] d \kappa
\end{aligned}
$$

which indeed satisfies both the differential equation (4) and the boundary condition (10), and is complex harmonic, in the form $f(z)+g(\bar{z})$ as expected.

Finally, we put the disturbance velocity potential in the following form:

$$
\Phi=R\left\{\ln \left|\frac{z-\zeta}{z-\zeta}\right|+G(x, y ; \xi, \eta)\right\} \exp (i \omega t) .
$$

2. Wave propagation. From the disturbance velocity potential (14), it is easy to see that the only term that contributes to the propagating wave is $G(x, y ; \xi, \eta)$ as given by (13). Now both integrals of $G$ are singular; principal values must therefore be considered.

The integrand of first.integral has singularities at the roots of equation

i.e., at

$$
\tau^{2} \kappa^{2}-[1-2 \tau(1-i \beta)] \nu \kappa+\nu^{2}(1-2 i \beta)=0
$$

$$
\kappa_{1,2}=\left[1-2 \tau(1-i \beta) \pm\left(1-4 \tau+4 \tau i \beta-4 \tau^{2} \beta^{2}\right)^{1 / 2}\right] \nu / 2 \tau^{2} .
$$

When $\beta=0$, these roots are

$$
\kappa_{1,2}=\left[1-2 \tau \pm(1-4 \tau)^{1 / 2}\right] \nu / 2 \tau^{2} .
$$

Similarly, the integrand of second integral has singularities at the roots of equation

i.e., at

\begin{tabular}{|c|c|c|c|c|c|c|c|}
\hline & \multicolumn{3}{|l|}{$\mathbb{R}$} & \multicolumn{4}{|c|}{$\mathfrak{g}$} \\
\hline & $\kappa_{1}$ & $\kappa_{3}$ & $\kappa_{4}$ & $\kappa_{1}$ & $\kappa_{2}$ & $\kappa_{3}$ & $\kappa_{4}$ \\
\hline $\begin{array}{c}0 \\
\rightarrow 1 / 4 \\
1 / 4 \rightarrow 1 / 2 \\
1 / 2 \rightarrow \infty\end{array}$ & $\begin{array}{cc}g / c^{2} & 0 \\
(+) & (+) \\
(+) & (+) \\
(-) & (-)\end{array}$ & $\begin{array}{c}g / c^{2} \\
(+) \\
(+) \\
(+) \\
(+)\end{array}$ & \begin{tabular}{l||}
0 \\
$(+)$ \\
$(+)$ \\
$(+)$
\end{tabular} & $\begin{array}{ll}0+ & 0 \\
0+ & 0 \\
(+) & ( \\
(+) & (\end{array}$ & $\begin{array}{l}0 \\
0+ \\
(-) \\
(-)\end{array}$ & $\begin{array}{l}0- \\
0- \\
0- \\
0-\end{array}$ & $\begin{array}{l}0 \\
0- \\
0- \\
0-\end{array}$ \\
\hline
\end{tabular}

$$
\tau^{2} \kappa^{2}-[1+2 \tau(1-i \beta)] \nu \kappa+\nu^{2}(1-2 i \beta)=0
$$

$$
\kappa_{3,4}=\left[1+2 \tau(1-i \beta) \pm\left(1+4 \tau-4 \tau i \beta-4 \tau^{2} \beta^{2}\right)^{1 / 2}\right] \nu / 2 \tau^{2} .
$$

When $\beta=0$, these roots are

$$
\kappa_{3,4}=\left[1+2 \tau \pm(1+4 \tau)^{1 / 2}\right] \nu / 2 \tau^{2} .
$$

TABLE I 
A careful investigation of (15) and (17) shows that as long as $\beta \neq 0$, the following results hold as $\beta$ approaches zero.

In the table, symbol $0 \pm$ indicates the following limiting behavior:

$$
\operatorname{Lim}_{\beta \rightarrow 0} g_{\kappa_{i}}=0
$$

As will be shown later, this limit physically corresponds to the case of an undamped propagation of a harmonic wave train of wave length $2 \pi / \kappa_{i}$. Indeed, by writing (13) in the form

$$
\begin{aligned}
G= & \frac{\nu}{\tau^{2}}\left[\int_{0}^{\infty} \frac{\exp [\kappa(y+\eta)]}{\left(\kappa-\kappa_{1}\right)\left(\kappa-\kappa_{2}\right)} \exp [-i \kappa(x-\xi)] d \kappa\right. \\
& \left.\quad+\int_{0}^{\infty} \frac{\exp [\kappa(y+\eta)]}{\left(\kappa-\kappa_{3}\right)\left(\kappa-\kappa_{4}\right)} \exp \left[i_{\kappa}(x-\xi)\right] d \kappa\right] \\
= & \frac{\nu}{(1-4 \tau)^{1 / 2}} \int_{0}^{\infty}\left(\frac{1}{\kappa-\kappa_{1}}-\frac{1}{\kappa-\kappa_{2}}\right) \exp [\kappa(y+\eta)] \exp \left[-i_{\kappa}(x-\xi)\right] d \kappa \\
& \quad+\frac{\nu}{(1+4 \tau)^{1 / 2}} \int_{0}^{\infty}\left(\frac{1}{\kappa-\kappa_{3}}-\frac{1}{\kappa-\kappa_{4}}\right) \exp [\kappa(y+\eta)] \exp [i \kappa(x-\xi)] d \kappa \\
= & \nu\left\{(1-4 \tau)^{-1 / 2} I_{1}+(1+4 \tau)^{-1 / 2} I_{2}\right\}
\end{aligned}
$$

and putting $\Omega=\kappa+i m$, all the integrals in (20) can readily be evaluated by proper choice of the contour of integration and application of Cauchy's integral theorem in the complex $\Omega$ plane. To fix the proper contour of integration, observe that, in order to make the integral meaningful, in addition to the necessary part of the positive real axis $(m=0,0<k<\infty)$ the remaining part of the contour must consist partly of the imaginary axis $(k=0$ ), (as the integral diverges along negative real axis due to the presence of the factor exp $[\kappa(y+\eta)]$ under the integral sign), and partly of a circular arc of infinite radius over which the integral vanishes. The choice of the proper quadrant for the contour $\Gamma_{i}$ for $I_{i}$ depends entirely on the sign of the difference $x-\xi$. When $x-\xi$

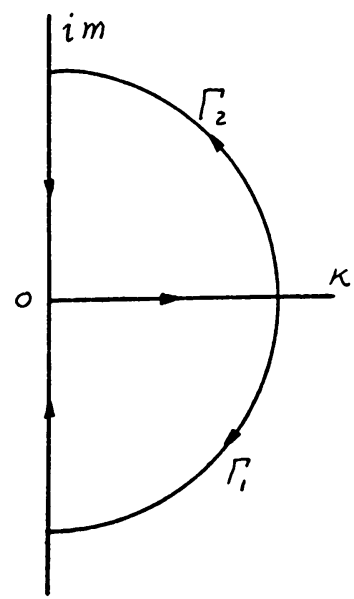

(a)

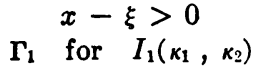

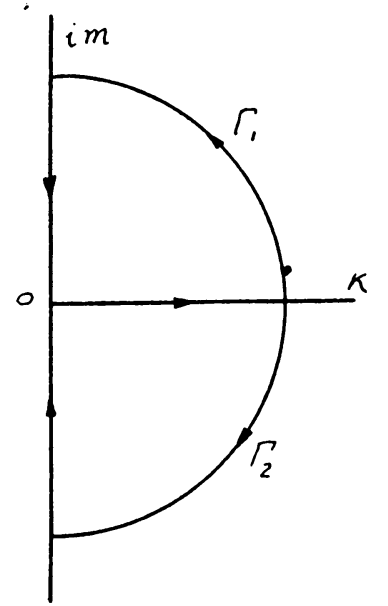

(b)

$$
\begin{array}{ll} 
& x-\xi<0 \\
\Gamma_{2} \text { for } I_{2}\left(\kappa_{3}, \kappa_{3}\right)
\end{array}
$$

FIG. 1. Contours of integration 
is negative, this part of the contour must be taken in the first quadrant of the complex $\Omega$ plane for $I_{1}$, and in the fourth quadrant for $I_{2}$, in order to insure convergence of the integral; and vice versa when $x-\xi$ is positive (see Fig. 1$)$. Whenever a pole $k_{i}(j=1,2$ for $I_{1}, j=3,4$ for $\left.I_{2}\right)$ lies inside its corresponding contour of integration $\Gamma_{i}(i=1,2)$, there will be a residue term by Cauchy's integral theorem. As will be shown later, this gives rise to a harmonic wave train which propagates in an undamped or damped manner, according to whether the pole is on or off the real axis, when $\beta$ approaches zero.

Taking cognisance of the above remarks, and applying Cauchy's integral theorem, we readily write the general expressions for the different cases as follows:

(a) $\underline{x-\xi}>0$

$$
\begin{aligned}
& I_{1}=\int_{0}^{\infty} \frac{\exp [\kappa(y+\eta)]}{\kappa-\kappa_{i}} \exp \left[-i_{\kappa}(x-\xi)\right] d \kappa \quad(j=1,2) \\
& =-2 \pi i \exp \left[\kappa_{j}(y+\eta)-i \kappa_{j}(x-\xi)\right] \\
& +\int_{0}^{\infty} \frac{\exp [-i m(y+\eta)]}{m-i_{\kappa_{i}}} \exp [-m(x-\xi)] d m, \\
& I_{2}=\int_{0}^{\infty} \frac{\exp [\kappa(y+\eta)]}{\kappa-\kappa_{i}} \exp \left[i_{\kappa}(x-\xi)\right] d \kappa \quad(j=3,4) \\
& =2 \pi i \exp \left[\kappa_{j}(y+\eta)+i \kappa_{j}(x-\xi)\right] \\
& +\int_{0}^{\infty} \frac{\exp [i m(y+\eta)]}{m+i \kappa_{i}} \exp [-m(x-\xi)] d m,
\end{aligned}
$$

(b) $\underline{x-\xi<0}$

$$
\begin{aligned}
I_{1}= & \int_{0}^{\infty} \frac{\exp [\kappa(y+\eta)]}{\kappa-\kappa_{i}} \exp \left[-i_{\kappa}(x-\xi)\right] d \kappa \quad(j=1,2) \\
= & 2 \pi i \exp \left[\kappa_{i}(y+\eta)-i \kappa_{j}(x-\xi)\right] \\
& \quad+\int_{0}^{\infty} \frac{\exp [i m(y+\eta)]}{m+i \kappa_{i}} \exp [m(x-\xi)] d m, \\
I_{2}= & \int_{0}^{\infty} \frac{\exp [\kappa(y+\eta)]}{\kappa-\kappa_{i}} \exp [i \kappa(x-\xi)] d \kappa \quad(j=3,4) \\
= & -2 \pi i \exp \left[\kappa_{i}(y+\eta)+i \kappa_{j}(x-\xi)\right] \quad \exp [m(x-\xi)] d m .
\end{aligned}
$$

It can be shown that all the definite integrals obtained above are regular, and they decrease rapidly with increasing $|x-\xi|$, hence they can only give rise to a local disturbance. Thus it is the residue term only that can lead to infinite propagation of harmonic wave trains. Indeed, noting that all the residues are given in the form

$$
2 \pi i \exp \left[\kappa_{i}(y+\eta) \pm i \kappa_{i}(x-\xi)\right]
$$

we can readily conclude that an undamped harmonic wave train appears whenever we 
have $\lim _{\beta \rightarrow 0} \mathscr{I} \kappa_{j}=0 \pm$. By further taking into account the detailed distribution of singularities in the various frequency ranges as listed in Table I, we obtain the following results:

(a) For $x-\xi>0$ (i.e. upstream of the travelling source):

$I_{2}$ : Since both $\kappa_{3}, \kappa_{4}$ lie outside of contour of integration $\Gamma_{2}$ for the entire range of parameter $\tau, I_{2}$ has no residue.

$I_{1}:$ i) for $\tau<1 / 4$, both $\kappa_{1}, \kappa_{2}$ lie outside of contour $\Gamma_{1}, I_{1}$ has no residue.

ii) for $1 / 4<\tau<1 / 2, \kappa_{2}$ lies inside $\Gamma_{1}$, so that $I_{1}$ has a residue term at $\kappa_{2}$. However, as $\lim _{\beta \rightarrow 0} g_{\kappa_{2}} \neq 0$, this residue term leads to a harmonic wave train that is damped out exponentially with $|x-\xi|$. For by writing $\kappa_{2}=m-i n$, where $n>0$, we have

$2 \pi i \exp \left[\kappa_{2}(y+\eta)-i \kappa_{2}(x-\xi)\right]$

$$
=2 \pi i \exp [m(y+\eta)-n(x-\xi)] \exp \{-i[m(x-\xi)+n(y+\eta)]\}
$$

which verifies our statement.

iii) for $1 / 2<\tau$, both $\kappa_{1}, \kappa_{2}$ lie outside of contour $\Gamma_{1}$, so that $I_{1}$ has no residue.

Thus, on the upstream side of the travelling source, the disturbance is always a local one.

(b) For $x-\xi<0$ (i.e. downstream of travelling source):

$I_{2}$ : For the entire range of $\tau$, both $\kappa_{3}$ and $\kappa_{4}$ lie inside the contour of integration $\Gamma_{2}$, so that $I_{2}$ has two residue terms at $\kappa_{3}$ and $\kappa_{4} . A s \lim _{\beta \rightarrow 0} \mathscr{S}_{3}, \kappa_{4}=0$, there will be two undamped harmonic wave trains of wave lengths $2 \pi / \kappa_{3}$ and $2 \pi / \kappa_{4}$ on downstream side which propagate to infinity. Indeed, it can easily be shown that

$$
\begin{aligned}
I_{2_{-}^{\prime}}^{*}=2 \pi i\left\{\operatorname { e x p } \left[\kappa_{4}(y+\eta)\right.\right. & \left.\left.+i \kappa_{4}(x-\xi)\right]-\exp \left[\kappa_{3}(y+\eta)+i \kappa_{3}(x-\xi)\right]\right\} \\
& +\int_{0}^{\infty}\left(\frac{1}{m-i \kappa_{3}}-\frac{1}{m-i \kappa_{4}}\right) \exp [-i m(y+\eta)+m(x-\xi)] d m
\end{aligned}
$$

which varifies our statement.

$I_{1}:$ i) for $\tau<1 / 4$, both $\kappa_{1}$ and $\kappa_{2}$ lie inside of contour $\Gamma_{1}$, so that $I_{1}$ has two residue terms at $\kappa_{1}$ and $\kappa_{2}$. As $\lim _{\beta \rightarrow 0} g_{\kappa_{1}}, \kappa_{2}=0$, two undamped harmonic wave trains of wave lengths $2 \pi / \kappa_{1}$, and $2 \pi / \kappa_{2}$ propagate to infinity on the downstream side. This can easily be seen as follows:

$$
\begin{aligned}
I_{1}=2 \pi i\left\{\operatorname { e x p } \left[\kappa_{1}(y+\eta)\right.\right. & \left.\left.-i \kappa_{1}(x-\xi)\right]-\exp \left[\kappa_{2}(y+\eta)-i \kappa_{2}(x-\xi)\right]\right\} \\
& +\int_{0}^{\infty}\left(\frac{1}{m+i \kappa_{1}}-\frac{1}{m+i \kappa_{2}}\right) \exp [i m(y+\eta)+m(x-\xi)] d m
\end{aligned}
$$

ii) for $1 / 4<\tau<1 / 2$, only $k_{1}$ lies inside the contour $\Gamma_{1}$, so $I_{1}$ has a single residue there. However, as $\lim _{\beta \rightarrow 0} \mathfrak{S} \kappa_{1} \neq 0$, the corresponding harmonic wave train is rapidly damped out.

iii) for $1 / 2<\tau$, both $\kappa_{1}$ and $\kappa_{2}$ lie outside of the contour $\Gamma_{1}$, so that $I_{1}$ has no residue.

Thus, on the downstream side of the travelling source, there appear multiple wave trains propagating to infinity. The number of these infinitely propagating wave trains is determined by the parameter $\tau$. 
The case of a travelling source of steady strength can easily be checked with Rayleigh's result. Here the poles are at $\kappa_{1}$ and $\kappa_{3}$ (Table I). Now, for points upstream, $x-\xi>0$, $\kappa_{1}$ lies outside the contour $\Gamma_{1}, \kappa_{3}$ outside $\Gamma_{2}$, so neither $I_{1}$ nor $I_{2}$ has any residue. For points downstream, $x-\xi<0$, however, both $\kappa_{1}$ and $\kappa_{3}$ lie inside their respective contours $\Gamma_{1}$ and $\Gamma_{2}$, and so both $I_{1}$ and $I_{2}$ have residues. As $\lim _{\beta \rightarrow 0} \mathscr{g}_{\kappa_{1}}, \kappa_{3}=0$, these residue terms represent undamped harmonic wave trains on the downstream side, of wave lengths $2 \pi / \kappa_{1}$ and $2 \pi / \kappa_{3}$ respectively. However, from Table I, we have $\kappa_{1}=\kappa_{3}=g / c^{2}$. Thus we only obtain a single harmonic wave train of wave length $2 \pi c^{2} / g$, as expected.

Summarizing, we have the following statements.

When a point source of constant strength travels with speed $c$ beneath the free surface of a two dimensional, ideal incompressible fluid of infinite depth and extent, aside from a local disturbance that travels together with the source, there is also produced a fundamental harmonic wave train of wave length $\lambda_{0}=2 \pi c^{2} / g$, or of frequency $\omega_{0}=g / c$, which propagates to infinity on the downstream side. There is no disturbance propagating to infinity on the upstream side. This was first observed by Rayleigh, and agrees exactly with the present analysis $(\tau=0)$.

When the strength of this travelling source fluctuates harmonically, then, aside from the local disturbance that travels with it, there are produced on the downstream side multiple wave trains propagating to infinity. This phenomenon may be regarded as a splitting of the original single wave for constant strength source by the fluctuation of source strength. The number of these infinitely propagating wave trains is determined by the value of a parameter $\tau$, which is the ratio between the fluctuation frequency $\omega$ of source strength and the frequency of fundamental wave $\omega_{0}$ explained above $\left(\tau=\omega / \omega_{0}\right)$. When $\tau<1 / 4$, there are four wave trains of different wave lengths propagating to infinity on the downstream side; when $\tau>1 / 4$, there are only two. No disturbance propagates to infinity on the upstream side.

Inspection of formula (20) reveals an interesting phase of the wave production by a travelling pulsating source. It is seen that the amplitude of both local disturbance and propagated wave trains contributed by the $I_{1}$-term exhibits a resonance behavior as the parameter $\tau$ approaches its critical value $\tau=1 / 4$. Thus violent disturbances should be expected at $\tau=1 / 4$ according to the present theory.

The present analysis also agrees with the physical intuition that no disturbance could propagate to infinity on the upstream side. This result can evidently be considered as a justification of our introducing the asymptotic upstream condition of "vanishing disturbance at infinity" as an alternative to Rayleigh's dissipative forces in rendering the solution unique.

\section{REFERENCES}

1. H. S. Tan, On source and vortex of fluctuating strength travelling beneath a free surface, Quart. Appl. Math. 13, (1955)

2. Lord Rayleigh, The form of standing waves on the surface of running water, Proc. Lond. Math. Soc. $15,69(1883)$

3. H. Lamb, On some cases of wave-motion on deep water, Ann. di Matematica 21, 237 (1913)

4. T. H. Havelock, The forces on a circular cylinder submerged in a uniform stream, Proc. Roy. Soc. A157, 526-534 (1936)

5. M. D. Haskind, The hydrodynamical theory of the oscillation of a ship in waves, Prinkladnaya Mat. Mekh. 10, 33-66 (1946) 